\title{
Commission 38
}

La Commission demande que le crédit annuel soit renouvelé à 4000 dollars.

\section{Commission 39}

La Commission 39 propose son ajournement jusqu'à ce qu'on ait besoin de la remettre en activité.

Elle demande au Comité exécutif de ne pas perdre de vue que le besoin d'un observatoire vraîment international continue de se faire sentir.

\section{Commission 40}

I. La Commission 40 recommande l'adoption de la résolution suivante: Considérant que la Radio Astronomie ne peut augmenter son importante contribution aux recherches solaires galactiques, et extra galactiques que si l'on peut trouver des bandes de fréquences libres de toute autre transmission, l'U.A.I. s'associe aux efforts de l'U.R.S.I. pour obtenir de telles bandes de fréquences. L'U.A.I. demande à l'U.R.S.I. de reprendre les négociations avec le C.C.I.R. pour obtenir des bandes de fréquences convenables, et attire l'attention de C.C.I.R. sur l'importance qu'elle attache à une action décisive de I'I.T.U.

2. La Commission 40 recommande que le Comité exécutif envisage la possibilité de tenir 'un symposium' de Radio Astronomie juste avant l'assemblée de Moscou de l'U.A.I. prévue pour I958. Elle suggère que ce symposium soit patronné à la fois par l'U.A.I. et l'U.R.S.I. Elle recommande qu'il se tienne de préférence en France, et qu'il dure une semaine.

3. La Commission 40 recommande l'adoption, pour la Radio Astronomie de la terminologie proposée par l'U.R.S.I. (cf. pp. $576-7$ de ce volume).

\section{Commissions 33 et 40}

Les Commissions 33 et 40 recommandent la création d'une sous-commission pour rechercher s'il est désirable de réviser la position du pole galactique et des longitudes galactiques. Elles suggèrent les nominations suivantes: Blaauw, Pawsey et Westerhout.

\section{Commission 42}

La Commission 42 de l'U.A.I. considère qu'il est hautement désirable que la publication de l'Observatoire de Cracovie, Ephémérides des binaires à éclipses, continue après la mort du regretté professeur TH. BANACHIEwicz. En effet, cette publication est du plus haut intérêt pour les observateurs d'étoiles variables à eclipses.

\section{Commissions 27 et 42}

Les Commissions 27 et 42 siégeant ensemble, affirment l'intérêt que présenterait la publication complète des observations photo-électriques originales des variables régulières (c'est-à-dire la magnitude instantanée avec le temps correspondant) au lieu des simples valeurs normales pour des phases données ou des représentations graphiques dont on se contente le plus souvent, et, en conséquence, chargent leurs Présidents d'informer de leur désir les éditeurs de journaux astronomiques et les directeurs d'observatoires, en sollicitant leur coopération. 